\title{
Phytochemical analysis of some plants from Lamiaceae family frequently used in folk medicine in Aligudarz region of Lorestan province
}

\author{
Gholamreza Asghari, Mohamad Akbari, Majid Asadi-Samani
}

\begin{abstract}
Many of the plants from the Lamiaceae family have been traditionally used as medicine all over the world. Also, some of the secondary metabolites isolated from this family have shown interesting biological function. In this study we have analyzed phytochemicals of some plants from Lamiaceae family frequently used in folk medicine in Aligudarz region of Lorestan province. In this regard, the plant species were collected and systematically identified during 2014-2015. The traditional and local uses of collected plants were questioned through informed consent semi-structured interviews with local informants. Phytochemical analysis was conducted to test the presence of compounds such as alkaloids, flavonoids, saponins, tannins,
\end{abstract}

anthraquinone and glycosides. 25 plant species belonging to 13 genes were collected and identified. The most uses of the plants were in treating cold, gastrointestinal disorders and as flavoring agents. From 25 plant species, a number of 23 species had tannin, 22 species exhibited positive reactions to flavonoids, 4 species showed positive reactions to alkaloids and 1 species exhibited positive reactions to saponins. This research has provided insights on the use of secondary metabolites in folk medicine for promotion of appropriate human health. The studied plants in this article can be seen as a potential source for discovering new drugs.

Keywords: Medicinal plants, Lamiaceae, Ethnobotany, Pharmacognosy.
Gholamreza Asghari, Mohamad Akbari

Department of Pharmacognosy, School of Pharmacy, Isfahan University of Medical Sciences, Isfahan, Iran

Majid Asadi-Samani

Student Research Committee, Shahrekord University of Medical Sciences, Shahrekord, Iran

Corresponding Author:

Mohamad Akbari

e-mail:pharmad144@yahoo.com

Accepted / Kabul: 01.03.2017
Submitted / Gönderilme: 26.12.2016

Revised / Düzeltme: 24.02 .2017

\section{INTRODUCTION:}

The study and identify of used medicinal plants by local people is desirable, not only for the discovery of therapeutic agents, but also because such information may be valuable for disclosing new sources of such economic materials as tannins, oils, gums, precursors for the synthesis of complex chemical substances, etc (1-5). In addition, the knowledge of the chemical constituents of plants would further be valuable in discovering the actual value of folkloric remedies (6-9). Medicinal plants are so important for healthy of individuals and communities for prevention and treatment of their diseases (10-14).

Many of the plants from the Lamiaceae have been traditionally used as medicine all over the world (15). The value of these plants lies in somechemical substances that produce a definite physiological action on the human body, especially through flavonoids and phenolic compounds (16). Many of these indigenous medicinal plants are used as spices and food plants (17). Also the Lamiaceae taxa have a significant role and are applied as infusion of the aerial parts or as powder 
mixed with honey or olive oil. The indigenous population also uses in folk-medicine derived from mineral and animal origin (18). Lamiaceae family with 46 genera and 410 species and subspecies have a great diversity and distribution in Iran. From these species, 124 species and subspecies (30\%) are endemic to Iran (19-21). Nepeta (76 sp.), Salvia (56 sp.), Stachys (34 sp.), Scutellaria (19 sp.), Phlomis (17 sp.), Eremostachys (16 sp.), Thymus (16 sp.) and Teucrium (12 sp.) are the largest genera in Iran (22). This family contains a wide variety of chemicals. Many species of the family have been experimentally studied and the efficiency of some traditional applications was confirmed by these researches. For example, the anti-bacterial characteristic of Thymus spp. (thyme) is due to the occurrence of thymol in this genus. Many other species such as Mentha spp. (mints) have also been shown to be antibacterial. These properties are due to the mono- and sesquiterpene in the essential oils of these plants, but, it is becoming increasingly apparent that other compounds (eg. diterpenoids) may be responsible (23). The therapy provided by the plant species could be attributed to the presence of wide arrangement of phytochemicals such as alkaloids, flavonoids, saponins, tannins, terpenoids and anthraquinone glycosides which have a curative activity against pathogens and therefore support their folklore medicinal plant (24-27).

Aligudarz is one of the most important geographic regions in Iran because of its ancient history of cultivating traditional medicinal plants. Some wild species of Lamiaceae family grow in this region. For this reason, they are used by the local herbalists for the treatment of various diseases and food additives. Thus, this study reviewed and analyzed the phytochemicals of plants from Lamiaceae family frequently used in folk medicine in Aligoudarz, Iran.

\section{MATERIALS AND METHODS}

\section{Geographic and climatic overview}

Aligoudarz is one of cities of the province of Lorestan in Iran, located 147 kilometers east of the center of the Lorestan. Its population is almost 142000 . Aligudarz city center mountainous have situated in the central Zagros Mountains, between $33^{\circ} 23^{\prime}$ northern altitude and $49^{\circ} 42^{\prime}$ eastern longitudes. The city's altitude is approximately 2000 meters above sea level. Aligoudarz region between $48^{\circ} 47^{\prime}$ and $50^{\circ} 03^{\prime}$ eastern latitudes and $33^{\circ} 44^{\prime}$ and $33^{\circ} 35^{\prime}$ northern longitudes with an altitude ranging almost from 1300 to 4050 meters. Annually, it rains $265 \mathrm{~mm}$ and the average temperature is $12^{\circ} \mathrm{C}$. District covers an area of approximately $5870 \mathrm{Km} 2$ (Figure 1).

\section{Preparation of herbarium species:}

In the present study, 25 plant species were collected in a proper growing season from plains, farms, and mountains from May

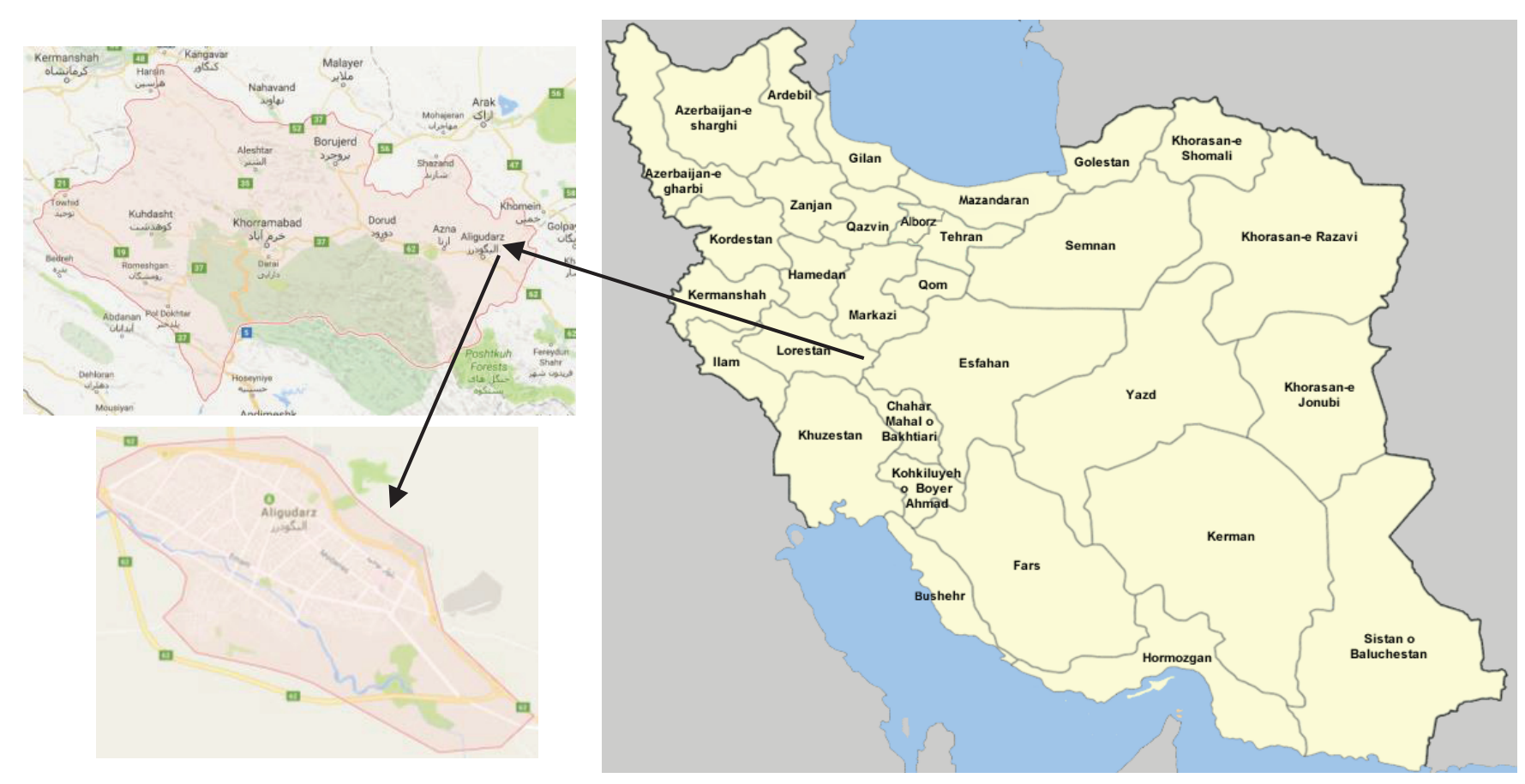

Figure1. Aligudarz region in Lorestan province of Iran. 
2014 to September 2015. After sample collection, the plants' species were dried and then pressed, and the samples with standard taxonomic were identified by Mr. Mohamad Taghi Fizi based on flora of Iran, Lamiaceae flora $(21,28)$, and medicinal plants and traditional medicine books $(29,30)$. The duplicates were intended for herbarium of Pharmacy and Pharmaceutical Sciences, Isfahan University of Medical Sciences, Isfahan, Iran.

\section{Data collection:}

In the first stage, samples were selected based on Morgan table (31). The sample size of local informants was 385 . This study was conducted among indigenous people of Aligudarz region. Of the 384 local informants (20 years and above) targeted population, 192 were interviewed using a questionnaire by informed consent. 192 of 384 harmonized information were used in this study.

Observation, oral semi-structured interviews and questionnaires were the tools commonly used for data collection. The plants were collected around the villages of the informants and were shown to them to confirm the plant names. The oral interviews were directed with the assistance of an interpreter, who was guided by village elders from different districts who were well introduced with the medicinal informants. The questionnaire contained information about local naming, parts which were used for medicinal-medicine-nutritional purposes and mode of folk preparation some of plants from Lamiaceae. All information was recorded. The data collection process, lasted 24 months.

\section{Procedure for phytochemical tests:}

Phytochemical analysis was conducted to test for the presence of compounds such as alkaloids, flavonoids, saponins, tannins, anthraquinone and glycosides. Arial parts of the 25 plant species of Lamiaceae collected from the study area were investigated. The chemical tests were carried out on the aqueous extract and on powdered sample from the arial part using standard procedures to identify the constituents as described by previous studies (32-38). The phytochemical screening of the various plant species was supposed to reveal the presence or absence of the various secondary metabolites (Table 1).

Table 1. Chemical basis and reagents for phytochemical tests

\begin{tabular}{|c|c|c|}
\hline Tests & Reagents & Positive result \\
\hline $\begin{array}{l}\text { Flavonoids } \\
\text { Shinoda's test }\end{array}$ & $\begin{array}{l}\text { Boric acid; Oxalic acid; } \\
\mathrm{HCl} \text { : } \mathrm{Zn} ; \mathrm{C}_{2} \mathrm{H}_{5} \mathrm{OH}\end{array}$ & $\begin{array}{l}\text { Strong red } \\
\text { At a wavelength of } 365 \mathrm{~nm} \\
\text { green/blue greenish yellow }\end{array}$ \\
\hline Alkaloid & $\begin{array}{l}\text { Mayer's reagent; } \\
\text { Wagner's reagent; } \\
\text { Dragendorff's reagent; } \\
\text { Potassium mercuric iodide } \\
\text { (iodine in potassium iodide } \\
\text { solution of potassium bismuth iodide) }\end{array}$ & $\begin{array}{l}\text { Orange ppt. } \\
\text { yellow colored precipitate } \\
\text { brown/reddish precipitate }\end{array}$ \\
\hline $\begin{array}{l}\text { Tannins } \\
\text { Braymer's test }\end{array}$ & $\begin{array}{l}\text { Gelatin; dist. } \mathrm{H}_{2} \mathrm{O} ; \mathrm{NaCl} \text {; } \\
\mathrm{FeCl}_{3}\end{array}$ & $\begin{array}{l}\text { Formation of jelly ppt. } \\
\text { Blue -black (hydrolysable) } \\
\text { Brownish-green (condensed/ nonhydrolysable) }\end{array}$ \\
\hline $\begin{array}{l}\text { Anthraquinones } \\
\text { Borntrager's test }\end{array}$ & Ammonia solution & Red coloration \\
\hline Glycosides & $\begin{array}{l}\text { Baljet-Reaction } \\
\text { Kedde-Reaction } \\
\mathrm{NaOH} \text { solution; Picric acid }\end{array}$ & $\begin{array}{l}\text { Orange ppt. } \\
\text { Purple coloration }\end{array}$ \\
\hline $\begin{array}{l}\text { Saponins } \\
\text { Froth test }\end{array}$ & $\mathrm{H}_{2} \mathrm{O}$ & Stable foam \\
\hline
\end{tabular}


Table 2. Medicinal plants used by the indigenous people of Aligudarz region

\begin{tabular}{|c|c|c|c|c|c|}
\hline $\begin{array}{l}\text { Scientific names } \\
\text { Voucher number }\end{array}$ & Local names & $\begin{array}{l}\text { Parts } \\
\text { Used }\end{array}$ & Local Usage & Persian names & Preparation \\
\hline $\begin{array}{l}\text { Ajuga chamaecistus Ging. ex Benth } \\
3100\end{array}$ & Unknown & L. & Analgesic & Moshkak sefis & Decoction; Poultice \\
\hline $\begin{array}{l}\text { Dracocephalum kotschyi Boiss } \\
3101\end{array}$ & Zar avi & L. & $\begin{array}{l}\text { Removing renal pain; Analgesic; } \\
\text { Tonic; Dyspepsia; Stomach ache; } \\
\text { Abdominal pain; Joints pain; } \\
\text { antitumor }\end{array}$ & Zarin giah & $\begin{array}{l}\text { Decoction; } \\
\text { Food additive }\end{array}$ \\
\hline $\begin{array}{l}\text { Eremostachys laevigata Bung } \\
\text { e } \\
3102\end{array}$ & $\begin{array}{l}\text { Kandal } \\
\text { Kouhi } \\
\text { Kangal }\end{array}$ & L.; R. & $\begin{array}{l}\text { relief of the pain } \\
\text { caused by insects } \\
\text { infection Female urinary tract }\end{array}$ & Gandna kouhi & Infusion; Poultice \\
\hline $\begin{array}{l}\text { Lamium amplexicaule L. } \\
3103\end{array}$ & Gazaneh sa & A.p & $\begin{array}{l}\text { Diarrhea; Diaphoretic; Anti } \\
\text { diabetics; Analgesic }\end{array}$ & Charkhak & Infusion; Poultice \\
\hline $\begin{array}{l}\text { Lamium album L. } \\
3104\end{array}$ & Gezgazo & A.p & $\begin{array}{l}\text { Diarrhea; Diaphoretic; Anti } \\
\text { diabetics; Analgesic }\end{array}$ & Gazaneh-sefid & Decoction \\
\hline $\begin{array}{l}\text { Lallemantia iberica F. \& C. M } \\
3105\end{array}$ & $\begin{array}{l}\text { Balangoo } \\
\text { shahri }\end{array}$ & S. & $\begin{array}{l}\text { Common cold; Stomach ache; } \\
\text { Coughing; Abdominal pain }\end{array}$ & Balangoo shahri & $\begin{array}{l}\text { Infusion; } \\
\text { Tea; } \\
\text { Soaked in water }\end{array}$ \\
\hline $\begin{array}{l}\text { Marrubium anisodon K.Koch. } \\
3106\end{array}$ & Gandna kouhi & $\begin{array}{l}\text { S.; } \\
\text { A.p. }\end{array}$ & $\begin{array}{l}\text { High blood pressure; Cardiac; } \\
\text { Common cold; Analgesic; Anti- } \\
\text { inflammatory; Pain caused by } \\
\text { insects Flatulence; Dyspepsia; } \\
\text { Women infertility }\end{array}$ & Ferasuon sefid & $\begin{array}{l}\text { Decoction; } \\
\text { Poultice }\end{array}$ \\
\hline $\begin{array}{l}\text { Mentha longifolia (L.) Hudson } \\
3107\end{array}$ & Pine & A.p. & $\begin{array}{l}\text { Flavoring agent; Dyspepsia; } \\
\text { Stomach tonic; Diarrhea; } \\
\text { Common cold; Infertility; Joints } \\
\text { pain; Dysmenorrhea; High blood } \\
\text { pressure; Coughing; Flatus; } \\
\text { Abdominal pain }\end{array}$ & Poneh & $\begin{array}{l}\text { Infusion; } \\
\text { Decoction }\end{array}$ \\
\hline $\begin{array}{l}\text { Phlomis olivieri Benth. } \\
3108\end{array}$ & Chlpo & Wh.p. & $\begin{array}{l}\text { Wound healing; Heart } \\
\text { Diseases; Gastrointestinal } \\
\text { diseases }\end{array}$ & Goshbare & $\begin{array}{l}\text { Poultice; } \\
\text { Decoction }\end{array}$ \\
\hline Salvia ceratophylla L.3109 & $\begin{array}{l}\text { Balbal } \\
\text { Goosh }\end{array}$ & A.p. & Common cold. & Maryam goli & Decoction \\
\hline $\begin{array}{l}\text { Salvia reuterana Boiss } \\
3110\end{array}$ & Shadona & Fr.; S. & $\begin{array}{l}\text { Abdominal pain; Edible; } \\
\text { Diarrhea; Eye pain; Depression }\end{array}$ & $\begin{array}{l}\text { Maryam goli-e- kouhi } \\
\text { Esfahani }\end{array}$ & $\begin{array}{l}\text { Decoction; } \\
\text { Edible }\end{array}$ \\
\hline
\end{tabular}




\begin{tabular}{|c|c|c|c|c|c|}
\hline $\begin{array}{l}\text { Salvia syrica L. } \\
3111\end{array}$ & Bala goosha & Fr.; A.p. & Edible abdominal pain & Maryam goli sori & Edible; Scorched \\
\hline $\begin{array}{l}\text { Salvia multicaulis Vahl } \\
3112\end{array}$ & $\begin{array}{l}\text { Earuneh } \\
\text { Bala }\end{array}$ & Fl. & Common cold; Migraine & Gol-earuneh & Decoction \\
\hline Salvia hydrangea DC. ex Benth3113 & Gol-earuneh & Fr.; A.p. & Common cold; Headache & Maryam goli & Decoction. \\
\hline $\begin{array}{l}\text { Salvia nemorosa L. } \\
3114\end{array}$ & $\begin{array}{l}\text { Bala } \\
\text { Gooshae }\end{array}$ & Fl. & Bile; Common cold. & Maryam goli & Decoction. \\
\hline $\begin{array}{l}\text { Stachys acerosa } \\
\text { Boiss } \\
3115\end{array}$ & Unknown & A.p. & Common cold. & Sonbole kohsari & Decoction \\
\hline $\begin{array}{l}\text { Stachys pilifera Benth. } \\
3116\end{array}$ & Korkegorbe & A.p & $\begin{array}{l}\text { Toothache; Edible; Tonic; } \\
\text { Analgesic; Edema; Expectorant; } \\
\text { tussive }\end{array}$ & Sonbole korkaloud & Decoction. \\
\hline $\begin{array}{l}\text { Stachys inflata Benth } \\
3117\end{array}$ & Olile & A.p & $\begin{array}{l}\text { Common cold; Analgesic; High } \\
\text { blood pressure. }\end{array}$ & Sonbole badkonaki & Decoction. \\
\hline $\begin{array}{l}\text { Stachys lavandulifolia Vahl } \\
3118\end{array}$ & Korkegorbe & A.p & $\begin{array}{l}\text { Headache, renal calculus } \\
\text { Common cold. Sedative } \\
\text { Flavoring agent. abdominal pain }\end{array}$ & Chay kouhi & $\begin{array}{l}\text { Decoction. } \\
\text { Food additive }\end{array}$ \\
\hline $\begin{array}{l}\text { Teucrium polium L. } \\
3119\end{array}$ & Chez & A.p & $\begin{array}{l}\text { Jaundice; Fever; Abdominal pain; } \\
\text { Diarrhea; Nausea; Blood sugar; } \\
\text { Respiratory distress }\end{array}$ & Kalporeh & Infusion \\
\hline $\begin{array}{l}\text { Thymus daenesis spp. daenensis } \\
\text { Celak } \\
3120\end{array}$ & $\begin{array}{l}\text { Oshom } \\
\text { orishom } \\
\text { mahali }\end{array}$ & Wh.p. & $\begin{array}{l}\text { Flatus; Fever; Kidney disorders; } \\
\text { Fever; Diuretic; Coughing; } \\
\text { Flavoring agent; } \\
\text { Antispasmodic }\end{array}$ & Avishan denai & $\begin{array}{l}\text { Food additive; } \\
\text { Decoction. }\end{array}$ \\
\hline $\begin{array}{l}\text { Thymus eriocalyx (Ronniger) Jalas } \\
3121\end{array}$ & Orishom & L. & $\begin{array}{l}\text { Flatus. Fever.Kidney disorders } \\
\text { Common cold. Asthma. } \\
\text { Antispasmodic }\end{array}$ & Avishan kouhi & $\begin{array}{l}\text { Food additive } \\
\text { Decoction. }\end{array}$ \\
\hline $\begin{array}{l}\text { Thymus lancifolius Celak } \\
3122\end{array}$ & Orishom & L. & $\begin{array}{l}\text { Common cold; Coughing; } \\
\text { Flavoring agent; Antispasmodic }\end{array}$ & Avishan kouhi & $\begin{array}{l}\text { Food additive; } \\
\text { Decoction. }\end{array}$ \\
\hline $\begin{array}{l}\text { Ziziphora clinopodioides Lam. } \\
3123\end{array}$ & Pine kouhi & A.p. & $\begin{array}{l}\text { Headache; Common cold; } \\
\text { Flavoring agent; Stomach ache; } \\
\text { Nausea; Appetizer; Stomach } \\
\text { tonic }\end{array}$ & $\begin{array}{l}\text { Kakuti Kouhi; Avishan } \\
\text { bargbarik }\end{array}$ & $\begin{array}{l}\text { Food additive } \\
\text { Decoction. }\end{array}$ \\
\hline $\begin{array}{l}\text { Ziziphora tenuior L. } \\
3124\end{array}$ & Pine kouhi & A.p. & $\begin{array}{l}\text { Appetizer; Stomach tonic; } \\
\text { Common cold }\end{array}$ & Kakuti; Pinah koei & $\begin{array}{l}\text { Food additive } \\
\text { Decoction. }\end{array}$ \\
\hline
\end{tabular}

A.p.= Aerial parts; S.= Seed; L.= Leave; Fl.=Flower; R.= Root; Rh. = Rhizome; Wh.p. $=$ Whole plant; Fr.=Fruit. 


\section{RESULTS}

A total of 25 plant species of Lamiaceae family collected from the study area were investigated. These plants belonged to 13 genera which were most commonly used by the indigenous people of Aligudarz region. The most parts of the plants that were frequently used by native people include aerial parts (52\%), leaf (20\%), flowers (16\%), seeds (12\%), roots (4\%) and whole plant (8\%).

In Aligudarz city, traditional medicines are mostly used to treat common cold and gastrointestinal disorders, although they are also used to treat variety of diseases. The information collected from folk medical practitioners were summarized and tabulated. Most of the researched plants were found to be very effective in the treatment of gastrointestinal infections and complications (Table 2).

From 25 plant species, a number of 23 species had tannin; 22 species exhibited positive reactions to flavonoid, 4 plants showed positive reaction to alkaloids and 1 plant species exhibited positive reactions to saponins. From about 25 plant species, certain phytochemicals combinations such glycosides and anthraquinon were absent among the studied species. The most frequent components were tannins and the least frequent were saponins (Table 3).

Table 3: Results of phytochemical tests/screening

\begin{tabular}{|c|c|c|c|c|c|}
\hline No. & Scientific name & Alkaloids & Flavonoids & Saponins & Tannins \\
\hline 1 & Ajuga chamaecistus Ging. ex Benth & + & - & - & + \\
\hline 2 & Dracocephalum kotschyi Boiss. & - & +++ & - & +++ \\
\hline 3 & Eremostachys laevigata Bunge & - & - & - & ++ \\
\hline 4 & Lallemantia iberica F. \& C. M & - & + & - & + \\
\hline 5 & Lamium album L. & - & + & - & + \\
\hline 6 & Lamium amplexicaule $\mathrm{L}$. & - & + & - & - \\
\hline 7 & Marrubium astracanicum Jacq. & + & ++ & - & + \\
\hline 8 & Mentha longifolia (L.) Hudson & + & ++ & - & +++ \\
\hline 9 & Phlomis olivieri Benth. & - & + & - & - \\
\hline 10 & Salvia nemorosa L. & - & + & - & ++ \\
\hline 11 & Salvia reuterana Boiss & - & + & - & + \\
\hline 12 & Salvia syrica L. & - & + & - & + \\
\hline 13 & Salvia ceratophylla L. & - & + & - & ++ \\
\hline 14 & Salvia multicaulis Vahl & - & + & - & + \\
\hline 15 & Salvia hydrangea DC. ex Benth. & - & + & - & + \\
\hline 16 & Stachys acerosa Boiss & - & + & - & + \\
\hline 17 & Stachys lavandulifolia Vahl & + & + & - & + \\
\hline 18 & Stachys pilifera Benth. & - & + & ++ & ++ \\
\hline 19 & Stachys inflata Benth. & - & + & - & ++ \\
\hline 20 & Teucrium polium L. & - & ++ & - & ++ \\
\hline 21 & Thymus daenesis spp. daenensis Celak & - & ++ & - & + \\
\hline 22 & Thymus eriocalyx (Ronniger) Jalas & - & ++ & - & ++ \\
\hline 23 & Thymus lancifolius Celak & - & + & - & + \\
\hline 24 & Ziziphora clinopodioides Lam. & - & ++ & - & + \\
\hline 25 & Ziziphora tenuior $\mathrm{L}$. & - & - & - & + \\
\hline
\end{tabular}

Key: + = Presences of constituents; - = Absence of constituents; $(+)$ score was recorded if the reagent produced only a slight opaqueness; $(++)$ score was recorded if a definite turbidity, but no flocculation was observed and $(+++)$ score was recorded if a definite heavy precipitate or flocculation was produced. 


\section{DISCUSSION}

This study introduced 25 plant species used by local people the Aligudarz, Iran. Although ancient sages through trial and error methods have developed herbal medicines, the reported uses of plant species do not certify their efficacy (39). Reports on ethnomedicinal uses of plant species required pharmacological screenings, chemical analysis, and the tests for their bioactive activities (40-43).

I this study, pharmacological screening of plant extracts provided an insight regarding both their therapeutic and toxic features. The presence of phytochemicals with various pharmacological and biological properties determines the medicinal values of various plant species as useful sources of drugs in ethnomedicine. These phytochemicals are the active ingredients in plants that make them useful in folk medicine $(44,45)$. These properties of plants have been confirmed in different trials (46-50).

Many species of the Lamiaceae family, especially endemics, are used locally by indigenous people in different parts of Iran. There is little information or documented references about their uses. For example, Phlomis olivieri Benth. L. is used locally in the Aligudarz region for wound healing and treatment of gastrointestinal diseases. Lamiaceae family is useful for scientists and pharmaceutical industries compared with other species. They can help us to identify medicinal plants as well as to eliminate the practices that may be harmful (51). Also the therapeutic effects of plants can be attributed to the phytochemicals such as alkaloids, flavonoids, sterols, saponins, tannins, terpenoids and glycosides which can show a curative activity against pathogens and therefore support their traditional usage in various illnesses $(52,53)$.

Alkaloids have analgesic, antispasmodic, antidiarrheal, and antibacterial properties (54-56). In the present study, some of the plant species with analgesic effects were Ajuga chamaecistus Ging. ex Benth, Marrubium astracanicum Jacq., Mentha longifolia (L.) Hudson, and Stachys lavandulifolia Vahl.

The flavonoids display a remarkable array of biochemical and pharmacological actions as well as anti-inflammatory, antioxidant, antiallergic, hepatoprotective, antithrombotic, antiviral antidiarrheal, and anti-carcinogenic activities (52, $56,57)$. In the present study, 22 out of the 25 plant species analyzed by exhibited qualitative methods had flavonoids. For example, Dracocephalum kotschyi Boiss. which contains flavonoids, is used in various illnesses including removing renal pain, analgesic; tonic seizure, dyspepsia, stomach ache, abdominal pain, Joints pain, and antitumor. Also it has been shown that thymus species have been used in folk medicine as antimicrobial, antispasmolytic, antibacterial, and analgesic agents due to the presence of their flavonoids (52).

Saponins are glycoside of both triterpenes and sterols and are used as expectorant and emulsifying agent (58-60). Stachys pilifera Benth is used in various illnesses including toothache, analgesic, edema, and tussive. Its extract was revealed to contain saponins and flavonoids which are known to produce inhibitory effects on inflammation.

Tannins have been reported to show antimicrobial, antitumor, antiinflamatory and wound healing properties in other organs (56). Moreover, tannins have been used for immediate relief of sore throats, diarrhea, dysentery, hemorrhaging, fatigue, and skin ulcers (61). Therefore, plant species from this study found to contain tannins which could have useful therapeutic aspects to cure various diseases. For example, Eremostachys laevigata is used for relief of the pain caused by insects and infection in female urinary tract.

\section{CONCLUSION}

According to our results, used plants in Aligudarz region can be as a potential source of useful drugs. Thus the evaluation of the pharmacological private for medicinal plants of this region is suggested and further researches on the secondary metabolites of the plants are recommended.

\section{Acknowledgments}

The authors would like to thank Dr. Seyed Ebrahim Sajjadi from Department of Pharmacognosy for helpful suggestions on this work. 
Lorestan ilinin Aligudarz bölgesinde halk ilacı olarak sıkça kullanılan Lamiaceae familyasından bazı bitkilerin fitokimyasal analizi

\section{ÖZ}

Lamiaceae familyasından birçok bitki tüm dünyada geleneksel halk ilacı olarak kullanılmaktadır. Bu familyadan hareketle izole edilen sekonder metabolitlerinde ilgi çekici biyolojik etkilere sahip olduğu gösterilmiştir. Çalışmamızda, Lorestan ilinin Aligudarz bölgesinde halkilacı olarak sıkça kullanılan Lamiaceae familyasından bazı bitkilerin fitokimyasal analizi yapılmıştır. $\mathrm{Bu}$ amaçla 2014-2015 yılları arasında bitkiler toplanmıș ve sistematik olarak tanımlanmıştır. Bitkilerin geleneksel ve bölgesel kullanılışları bölge halkından bilgilendirilmiş onam ve yarı yapılandırılmış görüşme yöntemiyle derlenmiştir. Bitkilerde; alkaloidler, flavonoidler, saponinler, taninler, antrakinon ve glikozitler gibi bileşenlerin varlığını saptamak için fitokimyasal analizler yapılmıştır. 13 Gene ait 25 bitki türü toplanmış ve tanımlanmıştır. Bitkilerin en çok, soğuk algınlığı ve gastrointestinal rahatsızlıkların tedavisinde ve aroma verici olarak kullanıldığı saptanmıştır. 25 Bitki türünden 23'ünün tanen içerdiği, 22 türün flavonoid, 4 türün alkaloid ve 1 türün saponin tanı tepkimeleri sonucunda pozitif sonuç verdiği tespit edilmiştir. $\mathrm{Bu}$ araştırma insan sağlığına katkıda bulunmak için geleneksel halk ilacı olarak kullanılan sekonder metabolitlerin kullanımı için bir yaklaşım sağlamaktadır. $\mathrm{Bu}$ makale kapsamında incelenen bitkiler, yeni ilaçlar keşfetmek için potansiyel birer kaynak olarak değerlendirilebilir.

Anahtar kelimeler: Tibbî bitkiler, Lamiaceae, Etnobotanik, Farmakognozi

\section{References:}

1. Baharvand-Ahmadi B, Bahmani M, Naghdi N, Saki K, Baharvand-Ahmadi S, Rafieian-Kopaei M. Medicinal plants used to treat infectious and non-infectious diseases of skin and skin appendages in city of Urmia, northwest Iran. Der Pharm Lett 2015; 7: 189-96.

2. Ghasemi Pirbalouti A, Momeni M, Bahmani M. Ethnobotanical study of medicinal plants used by Kurd tribe in Dehloran and Abdanan districts, Ilam province, Iran. Afr J Tradit Complement Altern Med 2013; 10: 368-85.

3. Ebrahimie M, Bahmani M, Shirzad H, Rafieian-Kopaei M, Saki $\mathrm{K}$. A review study on the effect of iranian herbal medicines on opioid withdrawal syndrome. J Evid Based Complementary Altern Med 2015; 20:302-9.

4. Bahmani M, Tajeddini P, Ezatpour B, Rafieian-Kopaei M, Naghdi N, Asadi-Samani M. Ethenobothanical study of medicinal plants against parasites detected in Shiraz, southern part of Iran. Der Pharm Lett. 2016;8:153-60.

5. Delfan B, Kazemeini H, Bahmani M. Identifying effective medicinal plants for cold in Lorestan province, west of Iran. J Evid Based Complementary Altern Med 2015; 20: 173-9.

6. Farnsworth NR. Biological and phytochemical screening of plants. J Pharm Sci 1966; 55: 225-76.

7. Bahmani M, Rafieian-Kopaei M. Medicinal plants and secondary metabolites for leech control. Asian Pacific J Trop Dis 2014; 4: 315-6.

8. Karamian R, Mohammadian A, Hassanimoghadam E, Alizadeh L, Abdali N, Keshvari M, Hosseini SM, Beyranvand $\mathrm{K}$. Identification and comparison of the yield and composition of essential oil constituents of four Eucalyptus species adapted to the climatic conditions of Khorramabad. J Herbmed Pharmacol 2015; 4: 25-8.

9. Bahmani M, Shirzad H, Rafieian S, Rafieian-Kopaei M. Silybum marianum: Beyond hepatoprotection. J Evid Based Complementary Altern Med 2015, 20: 292-301.

10. Asadi-Samani M, Rafieian-Kopaei M, Azimi N. Gundelia: A systematic review of medicinal and molecular perspective.
Pak J Biol Sci 2013; 16: 1238-47.

11. Akhlaghi M, Shanian Gh, Rafieian-Koupaei M, Parvin N, Saadat M, Akhlaghi M. Citrus aurantium Blossom and Preoperative Anxiety. Rev Bras Anestesiol 2011; 61:702-12.

12. Samarghandian S, Asadi-Samani M, Farkhondeh T, Bahmani M. Assessment the effect of saffron ethanolic extract (Crocus sativus L.) on oxidative damages in aged male rat liver. Der Pharma Lett 2016;8:283-90.

13. Asadi-Samani M, Kooti W, Aslani E, Shirzad H. A systematic review of Iran's medicinal plants with anticancer effects. J Evid Based Complementary Altern Med 2016;21:143-53.

14. Kooti W, Hasanzadeh-Noohi Z, Sharafi-Ahvazi N, AsadiSamani M, Ashtary-Larky D. Phytochemistry, pharmacology, and therapeutic uses of black seed (Nigella sativa). Chin J Nat Med 2016;14:732-45.

15. Naghibi F, Mosaddegh $M$, Mohammadi Motamed $M$, Ghorbani A. Labiatae Family in folk Medicine in Iran: from Ethnobotany to Pharmacology. Iran J Pharm Res 2010; 4:6379.

16. Tripathi I, Tripathi R, Tiwari A. Investigation of biologicaly active phytoconstituents present in selected plants material of Verbenaceae, Lamiaceae and Fabaceae family. Int Multidiscip Curr Res 2017;5: 31-7.

17. Nasri H, Baradaran A, Shirzad H, Rafieian-Kopaei M. New concepts in nutraceuticals as alternative for pharmaceuticals. Int J Prev Med 2014; 5: 1487-99.

18. Hendel N, Larous L, Sari M, Boudjelal A, Sarri Dj. Place of Labiates in folk medicine of the area of M'sila (Algeria). Glob J Res Med Plants Indig Med 2012; 1: 315-22.

19. Hedge IC. Labiatae of South-west Asia: Diversity, distribution and endemism. Proc R Soc Edinb, Section B: Biol Sci 1986; 89: 23-35.

20. Jamzad Z, Ingrouille M and Simmonds MSJ. Three new species of Nepeta (Lamiaceae) from Iran. Taxon 2003; 52: 93-8.

21. Labiatae. In: Flora Iranica, Vol. 150. Editors: Rechinger KH, Hedge IC, Ietswaart JH, Jalas J, Mennema J, Seybold S. Akademische Druck Verlagsanstalt, Graz. 1982. 
22. Naghibi F, Mosaddegh M, Mohammadi Motamed S, Ghorbani A. Labiatae family in folk medicine in Iran: From ethnobotany to pharmacology Iranian. J Pharm Res 2005; 2: 64-7.

23. Richardson P. The chemistry of the Labiatae: An introduction and overview. In: Advances in Labiatae Science. Editors: Harley RM, Reynolds T. Botanical Garden Kew. 1992.

24. Rafieian-Kopaei M, Asgary S, Adelnia A, Setorki M, Khazaei M, Kazemi S, Shamsi F. The effects of cornelian cherry on atherosclerosis and atherogenic factors in hypercholesterolemic rabbits. J Med Plants Res 2011; 5: 2670-6.

25. Khodadadi S, Rafieian-Kopaei M. Herbs, health and hazards; a nephrology viewpoint on current concepts and new trends. Ann Res Antioxid 2016; 1:e05.

26. Azadmehr A, Hajiaghaee R, Afshari A, Amirghofran Z, Refieian-Kopaei M, Yousofi Darani H, Shirzad H. Evaluation of in vivo immune response activity and in vitro anti-cancer effect by Scrophularia megalantha. J Med Plants Res 2011; 5: 2365-8.

27. Nasri H, Rafieian-Kopaei M. Tubular kidney protection by antioxidants. Iran J Public Health 2013; 42:1194-6.

28. Jamzad Z. Lamiaceae flora of Iran, NO 76. Research Institute of Forests and Rangelands. Islamic republic of Iran, 2012.

29. Heidari Sharifabad H, Vakili Shahrbabaki SA. Herbal Drugs and Traditional Medicine. Islamic Azad University Press of Jiroft. 2012.

30. Zargari A. Medicinal Plants, vol 5. Tehran University Press. 1992.

31. Small-Sample Techniques. The NEA Res Bull 1960; 38.

32. Harborne JB. Phytochemical methods. A Guid to Modern Techniques of Plant Analysis. Chapman \& Hall, London. 1998.

33. Townsend CC. Guest E, Al-Ravi A. 1966-1980 Flora of Iraq, Vol. 1-9. Ministry of Agriculture of the Republic of Iraq, Baqdad. 1998.

34. Markham KR. Techniques of flavonoids identification. Academic Press, New York. 1982.

35. Kapoor LD, Singh A, Kapoor SL, Srivastava SN. Survey of Indian plants for saponins, alkaloids and flavonoids. Lloydia 1969; 32: 297-304.

36. Mojab F, Kamalinejod M, Ghaderi N, Vahidipour HR. Phytochemical screening of Iranian plants. Iranian J Pharm Res 2003; 2: 77-82.

37. Salehi Surmaghi MH, Aynehchi Y, Amin GH, Mahmoodi Z. Survey of Iranian plants for saponins, alkaloids, flavonoids and tannins. Daru 1992; 2: 1-11.

38. World Health Organization. WHO guidelines for assessing quality of herbal medicines with reference to contaminants and residues, 2007.

39. Ahvazi M, Khalighi-Sigaroodi F, Charkhchiyan MM, Mojab F, Mozaffarian VA, Zakeri H. Introduction of medicinal plants species with the most traditional usage in Alamut region. Iran J Pharm Res 2012;11:185-94.

40. Nasri H, Abedi-Gheshlaghi Z, Rafieian-Kopaei M. Curcumin and kidney protection; current findings and new concepts. Acta Persica Pathophysiol 2016; 1:e01.

41. Cheraghi M, Asadi-Samani M. An overview of the most important medicinal plants affecting cardiac arrhythmia in Iran. Der Pharm Lett 2016; 8:87-93.

42. Madihi Y, Merrikhi A, Baradaran A, Rafieian-kopaei M, Shahinfard N, Ansari R, Shirzad H, Mesripour A. Impact of sumac on postprandial high-fat oxidative stress. Pak J Med Sci 2013; 29: 340-5.

43. Nasri H. Herbal drugs; from molecular studies to bedside investigations. Aria J Front Biochem 2017; 2(1):e01.

44. Jivad N, Asadi-Samani M, Moradi MT. The most important medicinal plants effective on migraine: A review of ethnobotanical studies in Iran. Der Pharm Chem 2016;8:462-6.

45. Jivad N, Bahmani M, Asadi-Samani M. A review of the most important medicinal plants effective on wound healing on ethnobotany evidence of Iran. Der Pharm Lett 2016;8:353-7.

46. Mohammadparast V. Antioxidant efficacy of Hibiscus esculentus. Front Biomed 2016;1:e04.

47. Bahmani M, Sarrafchi A, Shirzad H, Rafieian-Kopaei M. Autism: Pathophysiology and promising herbal remedies. Curr Pharm Des 2016; 22: 277-85.

48. Asadi-Samani M, Mousavi M, Baharara J. The synergic effect of low-frequency electromagnetic field and saffron extract on MMP gene expression in MCF-7 cell line. J Chem Pharm Res 2015;7:680-6.

49. Nasri H. Impact of garlic extract on platelet function and structure. Ann Res Platelets 2016; 1:e01.

50. Mirhosseini M, Baradaran A, Rafieian-Kopaei M. Anethum graveolens and hyperlipidemia: A randomized clinical trial. J Res Med Sci 2014;19: 758-61

51. Prance GT, Chadwick DJ, Marsh J. Ethnobotany and the search for new drugs. Wiley, Chichester. 1994.

52. Okach DO, Nyunja ARO, Opande G. Phytochemical screening of some wild plants from Lamiaceae and their role in traditional medicine in Uriri District-Kenya. Int J Herb Med 2013; 1: 13543.

53. Rafieian-Kopaie M, Baradaran A. Plants antioxidants: From laboratory to clinic. J Nephropathol 2013; 2: 152-3.

54. Stray F. The National Guide to Medicinal Herbs and Plants. Tiger Books International, London. 1998.

55. Rahimian GA, Rabiei Z, Tahmasebi B, Rafieian-Kopaei M, Ganji F, Rahimian R. Comparing the combined effect of garlic and mint extract with metronidazole in helicobacter pylori treatment. Iran J Pharm Sci 2013;9:63-7.

56. Hasoon A, Duraid O, Abbas AH. Comparative study of antidiarrheal effect of proposed new formulae from D. innoxia hydroalcoholic leave extract against E.coli O:157 induced diarrhea in goats. Kufa J Vet Med Sci 2015; 6: 56-66.

57. Middleton E, Andkandaswami C, Theoharides TC. The impact of plant flavonoids on mammalian biology: Implications for immunity, inflammation and cancer, in the flavonoids, advances in research science. Pharmacol Rev 2000; 52:673-751.

58. Uma C, Sekar KG. Phytochemical analysis of a folklore medicinal plant citrullus colocynthis L. J Pharmacogn Phytochem 2014; 2 : 199-200.

59. Hostettmann K, Marston A. Triterpene saponins pharmacological and biological properties. In: Pharmacology of Natural Products: Saponins. Cambridge University Press, New York. 1995.

60. Goze I, Alim A, Cetinus SA, Durmus N, Vural H, Goze HM. Chemical composition and antioxidant, antimicrobial, antispasmodic activities of essential oil of Thymus fallax Fisch Mey. J Med Plant Res 2009; 3:174-8.

61. Ashok PK, Upadhyaya K. Tannins are Astringent. J Pharmacogn Phytochem 2012; 1: 45-50. 